\title{
Cardiac Myeloid Sarcoma
}

National Cancer Institute

\section{Source}

National Cancer Institute. Cardiac Myeloid Sarcoma. NCI Thesaurus. Code C5370.

A rare extramedullary myeloid tumor that arises from the heart. It may present in association with or as a site of relapse of acute myeloid leukemia. Rare cases of myeloid sarcoma of the heart preceding acute myeloid leukemia have also been reported. 